Revue d'histoire de l'Amérique française

REVUE D.HISTOIRE DE L'AMÉRIQUE FRANÇAISE

\title{
Les Religieuses du Cap à Saint-Domingue (suite et fin)
}

\section{R. P. Cabon}

Volume 3, numéro 3, décembre 1949

URI : https://id.erudit.org/iderudit/801579ar

DOI : https://doi.org/10.7202/801579ar

Aller au sommaire du numéro

Éditeur(s)

Institut d'histoire de l'Amérique française

ISSN

0035-2357 (imprimé)

1492-1383 (numérique)

Découvrir la revue

Citer cet article

Cabon, R. P. (1949). Les Religieuses du Cap à Saint-Domingue (suite et fin).

Revue d'histoire de l'Amérique française, 3(3), 402-422.

https://doi.org/10.7202/801579ar d'utilisation que vous pouvez consulter en ligne.

https://apropos.erudit.org/fr/usagers/politique-dutilisation/ 


\section{LES RELIGIEUSES DU CAP Ã SAINT-DOMINGUE*}

(suite et fin)

Les religieuses et les négresses - Malgré toutes les objections et difficultés qu'on ne cessa de leur opposer, les Filles de Notre-Dame n'oublièrent jamais qu'un des buts de leur établissement à SaintDomingue était l'instruction religieuse, le relèvement moral et le soin matériel des pauvres négresses. A peu près toutes les religieuses s'y consacrèrent. Quelques-unes y eurent un mérite particulier, comme les mères Faulte du Puy du Tour, de Brivazac et La Brousse de Verteillac qui sortaient de familles de colons.

A côté du souvenir très spécial de la mère de Combalas "la bonne mère des Noirs", on a conservé celui de la Mère Gabriel Laurent d'Orfeuille, professe de Limoges, morte le 20 septembre 1780 après vingt-cinq ans de colonie. "L'œuvre qu'affectionnait surtout notre mère Laurans d'Orfeuille, c'était l'instruction des pauvres négresses, qui venaient trois fois la semaine dans les classes s'instruire des vérités de la religion. On sait combien ces pauvres créatures sont... malpropres et grossières. La patience de la mère d'Orfeuille obtint près d'elles de consolants résultats; elle en tira plusieurs du vice."

Deux anecdotes montreront à quelles natures les religieuses avaient affaire et à quels résultats elles étaient parvenues:

Le Père Aumônier prêchait la Passion pour les élèves noires, les blanches ne voulant point, par orgueil, assister à des offices communs aux unes et aux autres. Le prédicateur arrivait à la cinquième parole de Jésus mourant: "Sitio". Les négresses s'écrièrent d'une voix commune:

*Voir, pour premières parties de cet article : Revue d'Histoire de l'Amérique française, vol. II, no 4:557-575; vol. III no $1: 75-80$. On voudra bien se souvenir que cette étude a été rédigée par le P. Cabon, de la Congrégation du Saint-Esprit, sur des documents qui lui ont été fournis par le Dr Jouhaud, de Limoges. Le tout nous a été envoyé par M. Gabriel Debien. C'est par méprise que nous avons mis le nom de M. Debien au bas des deux premières tranches de cette notice historique. Nous nous en excusons auprès de notre correspondant. (Note de la Direction). 


\section{- Bon Dié a soif!} ça bon!

- Pauvre chai Bon Dié! Donné à Bon Dié du tafia avec citron,

Le prédicateur continuant: "Non, du fiel et du vinaigre".

Alors les enfants de sangloter et d'injurier en chœur les Juifs. ${ }^{2} 8$

On jugera à ce trait l'attachement que les noirs avaient pour les Religjeuses. Pendant les funérailles de l'une d'entre elles une des deux cloches de la sonnerie se fêla. Les noirs, par "gout d'imitation particulier à leur langue, se mirent à contrefaire le bizarre dialogue des sons, alternativement vibrants et rauques, résultat de cet accident. Un esclave adapta à l'air en guise de traduction, cette ritournelle qui à chaque nouveau glas de religieuse, faisait, parmi les noirs, d'un bout à l'autre de la ville, accompagnement au deuil général." 29

Les cœurs des négresses s'ouvrirent aux lumières de la foi. La mère Recoudert, de Limoges, qui avait succédé aux mères de Combolas, Laurent d'Orfeuille et de Mesonneyx, pouvait écrire au vicaire général de Limoges: "Notre Communauté est si nécessaire à la Colonie! Nos enfants font l'édification de tous. Les élèves nous sont extrêmement attachées; "et, parlant de la dévotion des négresses, elle concluait: "les blancs ne vont pas de pair". ${ }^{30}$

Après 1763 - Nous avons dit quels bouleversements subit la Colonie, le Nord surtout, après la Guerre de Səpt Ans: l'expulsion des Jésuites, leur remplacement par les Séculiers d'abord, puis par les Capucins, les troubles que causèrent ces changements, l'incertitude du statut ecclésiastique pendant quelques années et surtout l'altération de l'esprit public. Les Religieuses en souffrirent. Leur supérieur continua d'être le curé du Cap; plus tard le Préfet apostolique sollicita et obtint cette fonction; leur aumônier, approuvé par le Préfet, fut un séculier avant d'être un capucin; nous avons vu à cette charge, vers 1770 , l'abbé Belgarde, ancien missionnaire de Loango, en Afrique, et qui protesta à Rome contre les licences que se donnaient les Frères de la Charité.

Le couvent du Cap eut, à cette époque, la bonne fortune d'être gouverné par une Religieuse de foi solide et éclairée, dont le supériorat

28. Notice historique sur la Communaute des Religieuses Filles de Notre-Dame du Cap-Français (Saint-Domingue), 198.

29. Ibid., 187.

30. Ibid., 197. 
dura 14 ans, de 1768 à 1782, la Mère Marie-Aimée Lenoir de Mesonneyx, professe de Limoges.

Elle fut surtout attentive à préserver sa communauté de l'influence janséniste. L'esprit janséniste avait, en effet, réussi à se glisser dans quelques couvents des Filles de Notre-Dame, et ces couvents se montraient empressés à envoyer au dehors, quelques-uns de leurs membres afin de gagner des adeptes à l'hérésie; il semble bien pourtant que, en cela, ils étaient poussés par des gens haut placés, gagnés au jansénisme, plutôt qu'ils n'agissaient par leur propre mouvement. La Mère de Mesonneyx se mit en rapport avec les officiaux de quelques diocèses, hommes d'une doctrine sûre et d'une vertu éprouvée, pour qu'ils examinassent les Religieuses qui se présenteraient pour le Cap; elle aimait mieux manquer du personnel nécessaire que d'exposer ses Sœurs et ses élèves à la contagion de l'erreur. ${ }^{31}$

C'est sous le supériorat de la Mère de Mesonneyx que la communauté, par délibération du 20 juillet 1780, fut transférée à l'habitation Charrier au Haut du Cap, pour faire place aux troupes annoncées de $M$. de Guichen. Les autorités considérèrent, en face du dérangement imposé aux Religieuses, que les citoyens de la ville étaient déjà foulés par le logement des troupes, que tout autre arrangement grèverait les habitants autant que le trésor royal, que nulle part ailleurs les troupes ne pourraient être logées et contenues, avec plus de sécurité, puisque la maison était grillée et entourée de murs très élevés. En échange on offrait aux Religieuses la maison Charrier, "vaste, commode et décente où elles seroient en quelque sorte cloîtrées, cette maison étant entourée de murs, ainsi que les cours et jardins qui en dépendent, qu'elles y auraient une chapelle, le tout fermé par un portail et même grillé, s'il est besoin. L'administration s'engageait à faire constater légalement l'état de la maison conventuelle, avant que les

31. C'est peut-être d̀ ces difficultés intérieures que se rattache le retour en France de la sœur de Saint-Mandé du couvent d'Issoire. Elle était arrivée au Cap vers 1776. En 1782, elle-même et l'évêque de Clermont demandent son retour au ministre de la marine. L'évêque invoque les besoins de la maison d'Issoire. Le voyage aurait été retardé par la guerre. Malgré les représentations de la maison du Cap qui se voyait privée d'une religieuse dans un moment où nulle remplaçante ne pouvait arriver avant longtemps, il semble bien que l'entente ne fút pas parfaite et que ce départ fut ardemment désiré par la Mère de Saint-Mandé. (Arch. Nat. Colonies E. 303, dossier Saint-Mandé). 
troupes n'en prissent possession, et à la leur faire rendre dans le même état, aussitôt le départ des dites troupes". ${ }^{32}$

Durant le séjour de la Communauté à l'habitation Charrier, la Mère de Mesonneyx fut remplacée à la tête du Couvent par la Mère Delavaud, professe de Saint-Junien (1782).

Aucun renfort ne parvint tant que se prolongea la guerre; la paix faite en 1783, les couvents de France s'empressèrent de venir en aide à celui du Cap. Une lettre de Bordeaux l'annonçait aux Communautés. "Permettez-moi, disait la Supérieure, de profiter de cette occasion pour partager avec vous et votre Communauté, la sainte joie dont nous sommes toutes pénétrées par l'arrivée de quatre Religieuses de notra Ordre également distinguées par leur mérite, leurs talents et leurs vertus et qui vont au secours de l'établissement que nous avons à Saint-Domingue où l'on manque de sujets ${ }^{3} 3$ et où la moisson est abondante. C'est la Mère de Bonnefond, la Mère Laborderie, la Sœur de Goursaud, ancienne professe, toutes les trois de la Communauté de Limoges et la Sœur de Minard, encore novice, de celle de Périgueux. Elles sont ici pour s'embarquer et nous jouissons, en attendant, du spectacle si attendrissant pour nous, de voir nos respectables Sœurs se sacrifier pour la gloire de Dieu, renoncer généreusement à ce qu'elles ont de plus cher et braver les périls des mers pour aller se consacrer, le reste de leurs jours, à la pénible instruction de la jeunesse américaine".

La relation du voyage de ces religieuses nous a été conservée par l'abbé Legros qui avait été chargé de les accompagner de Limoges a Bordeaux. Il prit soin de recueillir les lettres qu'envoyèrent à leur

32. $\mathrm{Si}$, pour les Religieuses, la maison Charrier valait leur couvent, elle le valait aussi pour les troupes. Le seul motif plausible d'évincer les Religieuses de chez elles eât été la nécessité d'avoir toujours les troupes sous la main, en cas d'expédition pressée; ce motif, qui fut invoqué, aurait été justifié si les 2,000 hommes attendus de $M$. de Guichen avaient dû tenir garnison à Fort-Dauphin; il ne méritait pas d'être pris en considération lorsqu'il s'agissait de les loger au Haut du Cap; et en ce cas M. de Reynaud, gouverneur, n'évite pas le reproche de despotisme que voulait écarter son prédécesseur, M. d'Argout. On est tenté de supposer que l'administration militaire convoitait le terrain des Religieuses qui, avec le terrain tangent des Jésuites occupé par le Gouvernement, formait un ensemble très heureux: réuni, à celui des Grandes Casernes, il aurait permis, à l'ouest de la ville, un établissement militaire de haute importance. On s'empressa d'ailleurs d'y bâtir les Nouvelles Casernes et d'y faire d'autres travaux considérables.

33. Il n'y restait plus que cinq religieuses fort âgées. 
ancienne supérieure, en France, les sœurs arrivées au Cap. Nous ne résistons pas au plaisir d'en faire connaître de larges extraits. ${ }^{34}$

De la Mère Borderie

Le Cap, 24 février 1786.

Nous partímes de Bordeaux le jour de l'Immaculée Conception de la Très Sainte Vierge ${ }^{35}$ après avoir eu le bonheur d'entendre deux messes et nous être munies du Dieu fort. Nous montâmes en voiture. C'était une attention de M. Tabois, notre capitaine, qui voulut nous éviter une dizaine de lieues par eau, traversée pénible et quelquefois dangereuse. ${ }^{36}$

Après quatre jours d'attente au bord de la mer, le dimanche après avoir entendu la messe et communiê, nous nous embarquâmes sur une chaloupe, seules avec le capitaine, pour aller joindre le navire, à une distance de une lieue. Les autres passagers y étaient depuis huit jours, sans qu'on pût lever l'ancre à cause des vents contraires. Nous pouvons vous dire en toute vérité, que si on est exposé sur le navire pour son salut, c'est qu'on le veut bien. Tout est d'un grand ordre. Les messieurs sont fort retenus. Les matelots eux-mêmes n'ont pas dit un mot déplacé, sinon pendant les manceuvres où les commandants eux-mêmes sont obligés de proférer quelques jurements.

Il y a quatre principaux officiers, capitaine, sous-capitaine et deux lieutenants. Ils n'ont rien négligé pour nous faire apercevoir la différence qu'ils faisaient de notre état à celui des personnes du monde, quoiqu'il y eût deux dames et deux demoiselles parmi les passagers. Nous avons toujours oté distinguées à table et ailleurs.

Il faut bien ne pas oublier notre montée sur le gaillard qui n'est point aussi dangereuse ni désagréable comme on nous l'avait dite. Il y a là une echelle de bois de huit marches. On approche la chaloupe de manière que cela se fait très facilement. D'aussi loin qu'on nous aperçut on mit le pavillon, qui est une marque d'honneur. Nous étions seules avec M. Tabois... et tout le monde à notre arrivée criait d'une commune voix: Voici la chaloupe royale.

A peine arrivées à bord on nous conduisit dans le salon de compagnie où chacun vint nous saluer. La curiosité sans doute avait part à ces empressements. A côté du salon se trouvait la salle à manger fort jolie, mais pas ornée comme le salon qui avait tout autour des canapés rembourrés, les portes moitié vitrées avec des rideaux fort enjolivés d'ailleurs. Ce qui nous faisait le plus de plaisir, c'était une galerie à côté, où nous nous retirions souvent

34. Le texte de ces lettres est conservé dans le tome premier des Mélanges de l'abbé Legros, aujourd'hui aux Archives départementales de la Haute-Vienne, Fonds du Grand Séminaire de Limoges.

35. Soit le 8 décembre 1785. Elles avaient quitté Limoges le 3 novembre.

36. Les religieuses avaient $d \hat{a}$ attendre pour partir de Limoges une lettre du ministre de la Marine et la permission de l'évêque de Limoges. Leur passage était aux frais du roi. Il est donc probable qu'elles avaient été recommandées de haut aux bons soins du capitaine. 
pour faire nos prières, ce qui faisait dire: c'est l'église des religieuses. C'est là, ma chère Mère, que nous contemplions à loisir cette vaste mer, admirable ouvrage du Tout-Puissant.

Nous avions deux chambres petites, mais assez commodes, enjolivées au dehors de papier marbré et au dedans d'une peinture. Elles avaient chacune deux fenêtres et rideaux et fermaient à clef...

Nous voudrions continuer de vous dépeindre la construction du navire: mais comment nous y prendre. C'est une chose merveilleuse à voir. De vous parler des mâts, cela vous sera une énigme. La plupart [des navires] n'en a que deux. Le nôtre, qu'on nommait le Suply en avait trois. Ces mâts sont comme de grands arbres hauts de 160 pieds, d'une prodigieuse grosseur, peints, décorés, entourés de fers, avec une infinité de cordages formant des échelles, où les matelots sont obligés de grimper tel temps qu'il fasse... On y met jusqu'à trente-six voiles. La plus petite a autant de force pour conduire le navire que six paires de boeufs. Ces voiles sont de toile de demiaune: Il entre dans les plus grandes quatre cents aunes. A chaque instant on en met, on en ôte, selon que le vent est fort ou faible et cela avec une adresse admirable, en les montant par des poulies (ce) qui semble un amusement, à chaque fois, pour les matelots.

Le pont, c'est-à-dire le corps du navire, est de la longueur, comme serait depuis notre chœur jusqu'à la boulangerie, mieux planchéié que n'est le promenoir. Il est fort gracieux pour tout le monde d'avoir un endroit aussi vaste pour se promener. Il est au-dessus de toutes les chambres. Bien aéré comme vous pensez, il n'a aucune couverture. Après avoir passé le tropique on le couvre de tentes pour se garantir du soleil extrêmement chaud. Nous y arrivâmes le jour des Rois. Il nous semblait être au mois de juin. Alors nous avions fait 900 lieues, il nous en restait 700 à faire.

Ce navire a coûté à l'armateur trois cent mille livres. Il porte des provisions pour vendre, le montant de quatre fois autant: vin, farine ou autres choses manducables. Comme vous voyez, nous ne devions pas manquer, quand même la traversée aurait été de plusieurs années. On avait d'ailleurs fait la provision des passagers pour six mois.

Nous étions nourries supérieurement, c'est-à-dire en belles dames: nous le payerons bien dans la narration que nous voulons faire de notre maladie. Il y avait un maître d'Hôtel, cuisinier, domestique à tous nos petits soins.

Les repas étaient splendides. Ce qui nous a le plus étonnées était de manger du pain tendre trois fois la semaine, de la meilleure farine. On prenait le café, la liqueur après dîner. Je vous laisse à penser si dans cette recherche et dans bien d'autres nous avons négligé à faire apercevoir que nous étions des personnes religieuses, sans cependant aucune affectation, et cela a paru les édifier...

A coup sûr, l'enthousiasme des religieuses est d'abord fait da leur surprise. Elles redou taient la mauvaise éducation des gens de mer, 
les gestes risqués de l'embarquement! Au contraire ce ne sont que prévenances générales. Elles s'enchantent des manœuvres, du confort des cabines et de la table abondante, en bonnes Limousines, habituces au sol ferme et à la rude simplicité d'un ordinaire de couvent.

Ma chère mère, toutes ces belles et bonnes choses ne nous ont point empêché d'éprouver le mal de mer. La mère Bonnefond et sœur Borderie ont quitté ( ?) une barrique de bile! Cette maladie nous a assaillies dès le commencement de notre marche en pleine mer, c'est-à-dire le 19 décembre. Elle a dure quinze jours. Nous ne pouvions posseder aucune nourriture que du the ou de l'eau; encore ne les pouvions-nous garder. Le chirurgien major nous disait que pour guérir cette maladie, il fallait manger du biscuit. C'est un pain fait sans levain, et qui n'est point salé, il est ordinairement cuit d'une annee, ou pour le moins de six mois. Il est de la grandeur et épaisseur d'un fromage de deux sols. Quoique fait de la meilleure farine, il ne laisse pas d'être fort insipide pour des estomacs aussi dérangés qu'étaient les nôtres.

Le père $\mathrm{Blan}^{37}$ nous a parlé plusieurs fois de ce mal; mais il faut l'avoir éprouvé pour savoir à quel point de faiblesse il réduit. On est comme une personne qui a pris une forte dose d'émétique, et cela, tant que la maladie dure... Vous auriez eu compassion, mes chères mères, de voir la situation où cette maladie nous avait réduites... Il s'est operé un miracle en faveur de sceur Saint-Joseph. Elle n'a été atteinte en aucune façon, et nous a été d'un grand secours. Elle avait la peine de nous lever, de nous mettre au lit l'une après l'autre. Malgré ces grandes souffrances nous ne pouvions quelquefois nous empêcher de rire de notre piteuse situation.

Le navire faisait beaucoup de roulis. On avait eu l'attention d'assurer nos sièges, du moins notre siège, lorsque nous tombions, ne roulait pas sur nous. Le siège des autres, faute d'être arrêtés, ne manquaient pas de les suivre. Monsieur Roulis renversait les sauces. Ce n'est pas tout: la nuit nous entendions les passagers tomber de leurs lits. Nous en aurions fait autant si nous n'avions été retenues par des barrières.

Parlons du spirituel. Il nous a été libre d'observer après notre convalescence la règle depuis quatre heures jusqu'à neuf. Dieu merci, nous n'avons manqué aucun jeâne de précepte ni la règle tous les samedis. Monsieur Tabois nous prévint d'avertir le maître d'hôtel, chaque fois que nous voudrions faire maigre; et, en effet, ma chère mère, nous n'avons pas rougi, quoique nous ayons été seules à faire cet extraordinaire.

Les matelots nous ont édifíes en nous demandant des chapelets: nous leur en avons distribué quatre douzaines. Beaucoup ne le savaient plus dire. Ces pauvres gens venaient leur chapeau ou leur bonnet à la main nous prier de le leur apprendre. Vous jugez avec quelle satisfaction nous le faisions. Quelques-uns le portaient à leur cou, par dévotion. D'autres disaient qu'ils aimeraient mieux perdre 6 livres que leur chapelet.

37. Père Jacobin du couvent de Limoges. 
Nous étions confuses des attentions de ces gens-là. Aussitôt qu'ils voyaient que nous voulions nous promener sur le pont pour dire nos chapelets, ils écartaient les bois et les cordages. Ils se disaient contents d'avoir des religieuses et nous nommaient les femmes $d u$ Bon Dieu; c'est un terme dont ils se servent. Ils sont fort exacts à la prière qui se fait trois fois par jour; les offices sont chantés, mais tout cela sans prêtre.

Il faut vous faire part, ma chère mère, de notre vive douleur en nous voyant privées du P. Colomban ${ }^{38}$ qui était dans ce moment toute notre consolation et nous n'avions d'autres secours spirituels qu'un capitaine protestant... Ce ne fut point sa faute, si le P. Colomban ne partit pas avec nous; le capitaine ne trouvant pas de chambre telle qu'il la lui fallait, il fut oblige d'aller dans un autre (navire) et ne partit que huit jours après nous...

Si M. Vigne nous avait choisi un capitaine protestant, c'est qu'il lui reconnaissait une capacite extraordinaire pour conduire un vaisseau. Nous étions dans l'admiration de voir qu'il réunissait en lui toutes les qualités qui sont le propre d'un honnête homme. Il était bien malchanceux qu'il n'y joignit pas celle de catholique sans laquelle toutes les autres ne sont rien.

Il nous parlait souvent de la Sainte-Vierge et même nous chantait des Noëls, des cantiques. Il nous paraissait pénétré de la puissance de Dieu. Comme nous n'étions pas faites pour entrer en matière sur sa religion, nous crûmes qu'il valait beaucoup mieux par notre conduite, montrer l'exactitude que nous avions à la nôtre en la pratiquant dans toute sa rigueur; et en effet nous savons qu'il y a fait attention.

Nous oubliions souvent des livres de prières dans la salle de compagnie, mais volontairement, voyant que ces messieurs y faisaient des lectures avec plaisir, en disant qu'on gagnait indulgence à lire les livres des religieuses. M. Tabois 6tait fort jaloux de savoir les prières que nous faisions chaque jour, ne manquait pas non plus de les visiter. Il lut le saint sacrifice de la messe en français, tout entier, et nous dit après qu'à son âge, qui était de 35 ans, il n'en avait jamais autant fait, qu'il le trouvait très beau, étant surpris que les prêtres le disent en latin. Il prit occasion de nous parler de sa religion la veille de la Purification, jour auquel nous marquions la peine que nous ressentions d'être privées de la solenniser. Il nous avait promis en plaisantant un sermon, au cas où nous ne serions pas arrivés, ce que nous esperions beaucoup... Il nous parla longtemps de la religion protestante, mais sur toute chose, qu'on lui avait tiré trois fois son horoscope en lui disant toujours qu'il finirait par être moine. Il nous répondit qu'il ne se sentait point de vocation encore, mais que dans la suite ça pourrait bien être. Nous restâmes édifiées de sa façon de penser, nous aurions bien désiré le convaincre de la nôtre; il a fait souvent le sujet de nos réflexions. Nous le recommandons à vos prières. Nous n'avons qu'à nous louer de toutes les attentions et réserves qu'il a eues pour faire respecter notre état...

38. Capucin, originaire de Sarrelouis, qui était préfet apostolique, au Cap. Il s'était donné beaucoup de mouvement pour obtenir ces nouvelles religieuses. Il y avait intéressé le maréchal de Castrées, ministre de la Marine. En 1790 il était encore aumônier du Couvent. 
Enfin, ma chère mère, nous voici sur le point d'apercevoir la terre. Depuis quinze jours on faisait monter du matin au soir un pilotin au haut des mâts pour tâcher de la découvrir, et a chaque fois il nous disait toujours: demain, demain! A la fin, ce jour depuis longtemps si désiré arriva. Nous 6tions à faire notre oraison de quatre heures, admirant avec complaisance, le superbe élément et prévenant l'aurore qui commençait à paraître vers cinq heures; tout à coup nous entendîmes crier: Terre! Terre! qui mit la joie en tous les cœurs, aussi fortement que fait l'Alleluia le jour de Pâques, à des personnes qui ont fait carême dans toute sa rigueur, comme ferait la mère des Anges, ${ }^{39}$ qui sûrement le chante avec plaisir.

Cette terre n'était pas encore la promise, car elle était distante de 80 lieues. Son nom était Samana. ${ }^{40}$ Elle n'est habitée que par un seul hermite. Celle que nous vímes après était le Vieux Cap où il n'y a personne. La troisième fut enfin la nôtre, qui avait été désirée autant que celle de promission. Tout le monde se faisait un plaisir de nous l'annoncer. Jugez, nos chères mères, avec quelle joie nous voyions faire les preparatifs à jeter les ancres. Il nous semblait que cela hâtait le moment, ce moment dis-je, qui arriva deux jours plus tard, en nous privant de solemniser la fête de la Purification dans notre nouvelle maison. ${ }^{41}$

A peine eût-on arrêté le navire, que nous aperçûmes l'intendant de la communauté qui était chargé depuis un mois de s'informer à chaque bâtiment qui abordait si ce n'était pas le Sully... Il semblait que la trompette avait passé autour de la ville pour annoncer notre arrivée. Nos désirs étaient de passer en cognito (sic), mais il ne nous fut pas possible, car plusieurs officiers et messieurs de la ville vinrent à notre avance par une chaloupe, montèrent dans notre navire, nous firent mille honnêtetés et nous accompagnèrent avec une décence admirable. Pour sortir de la chaloupe, qu'on fait joindre le bord de terre, ce furent eux qui nous donnèrent la main pour monter et non pas les nègres, comme on nous avait plaisantées qui portaient. Nous n'avons point aperçu d'endroit qui fût dans le cas d'obliger à cela.

On débarque positivement au bord de la ville. Monsieur Noël ne fut pas le dernier à se rendre; il avait prétendu qu'il serait le premier, mais il perdit sa cause sur cet article. Il ne nous conviendrait pas de répéter les acclamations que nous eûmes en traversant la ville. Nous nous bornerons seulement à vous dire que nous ne pensâmes pas fendre la presse malgré les arrangements que ces messieurs avaient pris. Nous attribuons, ma chère mère, le plaisir que cette ville a témoigné à recevoir de pauvres étrangères au besoin qu'avait cette colonie de secours pour instruire des enfants qui ignoreraient toute leur vie ce que c'est que Dieu, sans cette communauté, à qui tous les habitants, jusqu'aux environs de quatre-vingts lieues, sont singulièrement attachés.

39. Mère Sénamaud, mère seconde de la maison de Limoges.

40. Sur la côte nord de la partie espagnole de Saint-Domingue.

41. Les Religieuses devaient débarquer le 4 février. 
Nous l'avons éprouvé par les visites continuelles que pendant huit jours nous avons reçues. A peine trouvions-nous le temps de prendre nos repas. Il y a deux parloirs; en sortant de l'un il fallait entrer dans l'autre, et toujours pleins jusqu'à la porte.

Vous pensez bien que les Capucins ne furent pas non plus les derniers. Ces pauvres nègres et mulâtres ne discontinuaient pas de nous crier: "Mâ̂tresses à moi! Maîtresses à moi!' C'était le seul langage que nous comprissions dans celui de créole. A présent nous avions (le texte) du catéchisme; nous comprenions encore ça et nous avions du plaisir à voir l'empressement qu'ils témoignaient à se faire instruire...

La jeune religieuse est encore étourdie de cette réception tout orientale. Elle a pris pour bel argent les manifestations d'une curiosité mondaine et d'une politesse à potentiel tropical. D'autre qualité furent les effusions des compagnes qui les attendaient.

Les quatre nouvelles ne trouvèrent au Cap que cinq anciennes dont elles font l'éloge:

Madame Lavaud, notre digne supérieure âgée de 73 ans, depuis bien des années a un cancer qui lui a rongé le sein et a gagné le côté et malgré cela réunit l'emploi d'infirmière, d'apothicaire, de portière, de sacristine. Y ayant été quinze ans, où elle a gagné plus de 30,000 livres, elle vient d'acheter une lampe pour l'église, en argent, qui lui coûte 5,000 livres, et 6 chandeliers également en argent, à 100 pistoles pièce. La sacristie est assortie pour le linge ou ornements à proportion. N'est-ce pas admirable que les doigts d'une personne aussi infirme aient pu faire tant de merveilles? Encore n'est-ce pas tout. Elle sonne tous les matins à quatre heures le lever, disant que les autres qui se portent bien ont besoin qu'on les ménage, que pour elle, qui étant déjà une patrague, [ elle ] ne risque rien. Quand les douleurs la pressent fortement, surtout en récréation, se voyant obligée de laisser son ouvrage, vous la verriez tout-à-coup dire; "Allons Lavaud, tu es une fainéante: ne sais-tu pas qu'il faut que la mort te trouve en chemin les armes à la main ?...

La mère Cases, ${ }^{42}$ fort aimable. Ses emplois sont (sic) seconde principale, qui forme les demoiselles à la piété et à une grande éducation. C'est un ordre en entrant au chœur et en sortant, qui vous enchanterait. Elle les fait toutes lire, chiffrer, travailler, les garde toute la journée, leur apprend le catéchisme et l'aumônier vient trois fois la semaine à la grille du chœur, pour leur en faire l'explication. Chacune à son tour se lève et je vous assure que cela les forme bien pour leur religion. Cet exercice se fait le soir à la place de l'oraison de demi-heure, en entendant l'instruction des demoiselles: C'est que les religieuses y trouve la leur.

La mère Recoudert est toujours telle que vous l'avez connue, fort charmante. On a, en vérité, rien à lui reprocher qu'un peu trop de timorité

42. Professe de Saint-Gaudens, Originaire de Vittoria, en Espagne. 
de conscience. Ne soyez done plus surprise qu'elle n'ait pas écrit à notre occasion. Elle est si détachée de tout qu'elle craindrait que la nature y eût part si elle s'en mêlait, disant qu'il faut que ce soit Dieu uniquement qui fasse cet ouvrage. Elle ne veut point de connaissance et ne va peint au parloir que pour son emploi, qui est de procureuse, fait écrire des demoiselles, va les garder pendant qu'elles dansent...

La mère Sarrieux est un caractère à souhait, de la plus grande douceur et prévenante à toutes. Elle est de la maison de Saint-Gaudens... Son emploi est aux classes avec la Mère Marais, joint la lingerie qui n'est pas un petit morceau, à raison de la quantité de linge que consomment les sueurs abondantes qu'on a dans ce pays.

Pour la mère Marais, vous serez charmée d'apprendre qu'elle donnait la préférence à la communauté de Limoges, si toutefois elles avaient été obligées de passer en France dans leur désastre comme vous verrez dans la relation qu'elle a bien voulu se donner la peine d'écrire sachant que cela vous fera plaisir... Pour les talents, elle les a tous réunis en elle, étant âgée de 29 ans et ayant pris l'habit ici. Comme vous voyez, elle aurait droit de nous mettre toutes dehors, étant la seule native de cette communauté.

Quoique peu nombreuses, elles ont toujours observé la règle dans tous ses points, excepté du dîner qui ne se fait qu'à onze heures, à raison de la viande qu'on ne peut avoir que très tard. Le vendredi et le samedi sont comme il est prescrit, n'ayant pas cette difficulte. L'oraison du matin se fait de cinq quarts d'heure d'horloge; l'examen d'avant le diner se fait d'un quart d'heure exact, et le reste comme à Limoges; il est vrai qu'à raison des emplois qui nous dispersent il n'est pas possible de dire l'office en psalmodiant, mais on le sonne exactement, afin que celles qui [ le ] peuvent, se rendent et le disent, chacune à leur particulier, tout bas...

La maison est très vaste, bien aérée. Elle est placée au milieu de deux jardins garnis d'orangiers qui nous embaument, nos chambres très jolies, mais dans la plus grande simplicité... Il n'y entre que trois [ nègres ] tous les jours pour le jardin, et ressortent exactement tous les soirs. Il est vrai qu'elles ont onze négresses pour les besoins de la maison; il y en a eu jusqu'ici destinées à servir au réfectoire. Voyant le peu qu'elles étaient, maintenant nous prenons avec plaisir leurs places...

Les voilà done bannies de cet endroit. Nous ne pouvons pas en faire autant de la cuisine, en les remplaçant par des sœurs converses, le Roy étant entièrement opposé qu'on n'en reçoive pas, alléguant que nous ne manquons pas de personnes pour être servies, d'ailleurs que c'est un moyen de donner du pain à des pauvres filles qui n'en ont pas.

Avant de finir, nous voulons vous parler des secours spirituels qui nous ont surprises agréablement. Depuis la destruction des Jésuites, messieurrs les Capucins, gens très savants et fort zélés pour la gloire de Dieu, sont aux points nommés de la Communauté, comme ce qu'étaient autrefois les premiers missionnaires... Je conviens qu'il en coûte cher, mais on ne regrette rien dès qu'il s'agit de se procurer les secours spirituels. On donne à l'au- 
mônier, qui est aussi confesseur, tous les ans 2000 livres, une maison à côté de la nôtre. Joint à cela on le blanchit, on le nourrit et il a droit d'envoyer chercher tout ce qui lui fait plaisir. On est encore obligé de payer les médecins et les remèdes s'il est malade.

Il est vrai que la communauté, comme on vous avait dit, est riche. Elle en a besoin, vu les dépenses considérables qu'on est continuellement obligé de faire...

Nous voyons ici des choses que vous aurez peine à croire: c'est que nous mangeons des pommes aussi souvent qu'à Limoges. On nous assure qu'on trouve les mêmes fruits au marché qu'à Bordeaux, jusqu'à des cerises frấches, des châtaignes, et $M$. de Seilhac nous a assuré qu'il nous enverrait les premières qui paraîtraient. Je vous laisse à penser si ces sortes de choses nous affectent...

Nous ne parlons pas de nos emplois; nous ne les aurons que lundi prochain, première semaine de Carême. Jugez si on nous a fait reposer! Nous avons pensé que vous verriez avec plaisir une petite relation des aventures qui étaient arrivées à nos religieuses dans le temps de la guerre. Nous vous l'envoyons plus volontiers étant persuadées que M. Vigne payera le port et qu'il ne vous en coûtera rien, pas plus qu'à l'avenir.

Nous serons en Amérique, comme nous étions en Europe, toujours dans les sentiments d'affection la plus sincère, d'amitiê la plus tendre et ceux du profond respect avec lequel nous avons l'honneur d'être...

La lettre de la mère de Bonnefond à sa sœur religieuse du couvent de Limoges est d'un moindre intérêt. Ce n'est plus la verve à la fois naïve et enjouée de la mère Borderie. L'âge ne doit pas être le même. Il y paraît au style, moins alerte. Son esprit va aux réalités immédiates. Tout d'abord quelques détails sur la traversée; il est question du navire.

De notre maison du Cap, 24 février 1786.

C'est étonnant le bruit qui se fait dans cette merveilleuse habitation est incompréhensible... le tapage étant égal en tout temps étant malade, ne pouvant reposer ni jour ni nuit... Il y avait aussi un comédien et sa femme qui ont été de la plus grande retenue, que ce soit à cause de nous ou non. D'ailleurs nous n'avions pas grand chose à démêler avec eux. Pour les autres passagers c'était quelques jeunes gens que nous n'avons presque pas vus ni parless, excepté un qui mangeait à la table du capitaine. Il avait une éducation admirable et toutes sortes d'attentions pour nous. Tous les officiers les ont portées à un point qu'il serait difficile de vous exprimer.

Il ne s'est attrapé qu'un poisson pendant la traversée. Ils en étaient désolés, à cause de nous, disaient-ils, et du maigre que nous faisions...

Quand nous abordâmes pour mouiller, c'est-à-dire arrêter pour descendre, il sembla que c'était une forêt [ de mâts ]...

Mais les descriptions ne sont point le fort de la Mère Bonnefond. Elle passe vite à des jugements de bonne ménagère. 
On garde pourtant les règles de la modération par esprit religieux. La nourriture est telle: le pain tous les jours tendre et beaucoup meilleur que le vôtre, le vin excellent, à discrétion: on ne risque rien, celles qui en boivent le plus [ en prennent] un demi-verre. On n'a pas besoin de s'échauffer; la soupe excellente soir et matin; le bouilli: bon bœuf veau ou mouton, un autre plat de viande, volaille, etc... Toujours au matin, de la salade et deux ou trois sortes de légumes, petits pois verts, haricots, artichauts, asperges, enfin tout ce qu'on peut avoir dans le printemps est ici on toutes saisons. Il faut convenir que cela n'a pas autant de goût et de substance; c'est pour cette raison qu'on multiplie le nombre à chaque repas. Le soir, jamais de viande, un potage, un œuf frais, du riz ou millet au lait ou à l'eau avec des légumes. Les fèves blanches et rouges n'y sont point inconnues. Tu sais que je les aime.

Il y a grand nombre de fruits, tous insipides, exceptés l'orange, la grenadine qui ressemble un peu au melon, de goût différent et meilleur. La sapotille a du rapport au brignon. Tous les autres sont si fades qu'ils vous porteraient au cour.

Le réfectoire est fort joli, surtout la chaire pour lire. La lecture se fait comme chez nous et le Benedicite. Il y a trois grands lustres de verre à l'infirmerie et à la salle, de semblables pour s'éclairer et conserver la lumière qui est considérable surtout les soirs.

Il y a plus de 100 fenêtres dans la maison pour que l'air y pénètre bien, toutes sans vitres, vu les tremblements de terre qu'il y a quelquefois. Cela m'étonne que les religieuses soient servies en faïence. Elles n'ont rien en étain.

Le chœur a du rapport à celui de Bordeaux. Il y a un autel de la Sainte Vierge où la statue qui la représente est de toute beauté: huit cierges y brâlent continuellement. C'est la dévotion des nègres - pour celles des blancs elle ne va pas de pair. - Il ne leur manque à ces pauvres gens que l'instruction...

Les onze négresses qui sont au service de la maison aident beaucoup dans les emplois. Il y en a une qui reste toujours à la porte, qui avertit les religieuses et demoiselles. Il n'y a point de cloche pour cela. Par conséquent point de signaux. Une autre à l'apothicairerie, deux à la sacristie, plusieurs à la cuisine. On a une entière liberté pour les commander. Elles volent pour le faire. Elles sont pieds nus, mais d'ailleurs mises décemment. Il y en a qui n'ont qu'une jupe, un grand mouchoir tendu sur les épaules et un qui leur plie (sic) la tête. C'est la coiffure ordinaire de ces pays, surtout de ces gens. D'autres ont des casaquins avec la jupe; quoiqu'esclaves, ils sont de mousseline rayee ou de belle indienne.

Les pensionnaires sont de même. Il y en a qui en ont [des robes] de linon gazé, et par-dessous une jupe de taffetas de couleur. La modestie des demoiselles est admirable. Toutes ont de grands mouchoirs, jusqu'aux plus petites, la plupart en bonnet rond de mousseline ou grand bonnet de gaze. On les instruit fort bien. On en exige même plus qu'en France pour leur première communion. C'est incroyable comme elles peuvent suffire 
a tout. Celles des classes sont très bien aussi. Je peux assurer que je n'en ai laissé aucune dans les vôtres aussi avancée pour la lecture et l'écriture... C'est dans ces sentiments et ceux de la plus tendre affection, que je me dis, chère Sœur...

Appréciation générale. - Le lecteur se méfiera peut-être du ton louangeur des Religieuses les unes à l'égard des autres, soit de celui des Supérieures dans les notices des défuntes, soit de celui des Sœurs qui débarquent et jugent à première vue les anciennes de la Communauté. Volontiers, nous admettons que, sous ces qualités qu'on prône, se cachent parfois des défauts oubliés avec le temps et comme effacés par une sainte mort, ou qu'on n'aperçoit qu'après une longue expérience: on en agit ainsi dans toutes les Communautés; mais on ne saurait pourtant mettre en doute la bonne foi de ces correspondances.

Une observation s'impose. Quand les éloges ainsi distribués n'ont rien de déterminé mais restent indécis dans leur généralité, on peut sans doute en rabattre; il n'en est pas de même quand ils visent une vertu particulière. Dans ce cas, l'affection ou la vénération n'aveuglent pas à ce point qu'elles fassent attribuer une qualité définie à qui ne la possède pas à un degré remarquable. Cette qualité n'exclut sans doute que le travers contraire et non les autres; mais il est des vertus qui transforment un caractère au point d'ən bannir beaucoup de défectuosités: la vertu dominante s'insinue dans tous les rouages de l'âme et purifie celle-ci de nombreuses scories; tel nous semble avoir été le cas de la plupart des Religieuses que nous avons vantées sur le témoignage de leurs contemporaines; si leur portrait, tracé par leurs Sœurs, met en relief un beau côté de leur âme, ce relief est assez puissant pour donner à l'ensemble une beauté morale d'ordre supérieur.

Il nous reste à reproduire ici l'appréciation de Moreau de SaintMéry sur le couvent du Cap. C'est l'opinion d'un magistrat créole. Elle est partagée, on peut le croire, par la majorité de sa classe, classa moyenne de gens qui ne possèdent pas la fortune des grands habitants sucriers, qui en montrent souvent la morgue et qui mesurent avec soin la distance qui les sépare de leurs inférieurs.

L'établissement des Religieuses a de grands désapprobateurs et l'on en a une preuve évidente dans le petit nombre de leurs pensionnaires qui ne va guère à présent (1789) que de quarante à cinquante, tandis qu'elles en avaient davantage, il y a soixante ans. Cette désapprobation, il faut le dire, a pour cause quelques exemples de relâchement dans la surveillance de ces pensionnaires et plus encore de ce qu'elles en ont qui ne sont pas des 
blanches. D'ailleurs l'éducation qu'elles procurent est toujours incomplète du côté des talents agréables, et quant à moi, j'inclinerai toujours pour que nos jeunes créoles soient élevées en France, parce qu'elles y sont à l'abri du despotisme dont le service des esclaves leur donne l'habitude et le goût. Mais il faut cependant que ce ne soit qu'autant qu'on pourra s'assurer qu'elles y auront des soins capables de les sauver des dangers d'un pays où les mœurs exigent la plus grande vigilance et de celui de s'entendre exagérer sans cesse leur fortune.

On aura vite fait de réduire à leurs justes proportions les griefs de la société créole contre les Religieuses. Voici en outre quelques notes sur la composition de la maison.

Le couvent du Cap peut encore être très utile dans beaucoup d'autres cas, dans ceux, trop communs, par exemple, où de seconds mariages donnent des marâtres à de jeunes personnes qu'il faut affranchir de cette domination. - Les Religieuses ont sept classes; quatre pour leurs pensionnaires, trois autres où cent petites filles de la ville apprennent gratuitement à lire, à écrire et l'arithmétique. Trois fois par semaine, il y a des instructions publiques pour les devoirs religieux. Chaque année les pensionnaires donnent un exercice public.

Au sujet du revenu de la Communauté, Moreau de Saint-Méry déclare ne pas le connaître exactement mais il sait qu'il suffit à la dépense.

La Communauté a reçu plusieurs dons. Le Conseil du Cap lui a accordé le 11 décembre 1734, 30,000 livres sur un legs de M. Colleno. Le même tribunal a homologué, le 6 juillet 1743, une donation de 10,000 livres faite par Mme Le Tellier, le 27 juin 1742, pour en aider les bâtisses, à la charge de loger, nourrir, vêtir et élever à perpétuité une jeune fille orpheline et nécessiteuse, au choix de la donatrice et de ses successeurs. M. le Comte d'Héricourt a aussi fondé à perpétuité une messe de requiem, tous les mercredis, dans la chapelle des Religieuses, pour le repos de l'âme de son épouse. M. Barraut leur a légué, en 1758, 2,000 livres pour procurer des ornements à leur chapelle". Enfin divers détails: "Quoique le syndic doive être renouvelé tous les trois ans, celui actuel a bien prolongé son exercice, car il l'était déjà en 1772. Pour faire une signification à la supérieure des Religieuses, l'huissier prend une permission par écrit du Procureur général. Ce magistrat et les Administrateurs de la Colonie se sont arrogé le droit d'entrer quelquefois dans l'intérieur du couvent et toujours avec une suite nombreuse: je doute que cela soit ni utile, ni décent. Les Religieuses de Notre-Dame, qu'on appelle aussi les Filles Sainte Marie, portent un habit noir, une guimpe blanche et un voile noir. ${ }^{43}$

43. Moreau de Saint-Méry, I: 432. 
L'agonie d'un couvent. - Dès la fin de 1789 la Colonie commença à s'agiter. A ce moment la Communauté des Religieuses avait perdu sa supérieure; elle avait succombé à son mal; en 1791, la Mère de Sarrieu devait retourner en France; nous ignorons si d'autres Mères vinrent tenir la place des disparues; il semble bien qu'une novice au moins, originaire du Cap, la Mère de Saint-Martin, fut dès ce temps agrégée au couvent.

Un premier incident attira l'attention sur les Religieuses. On sait qu'au mois d'avril 1790 se réunit à Saint-Marc une Assemblée des représentants de la Colonie entière, qui se donna mission de voter les Bases Constitutionnelles de Saint-Domingue. Entre temps elle recevait, après les cahiers des paroisses, les doléances de tous ceux qui avaient à se plaindre des abus d'autorité d'où qu'ils vinssent. Dans la séance du 18 mai, au dépouillement du courrier du Nord, fut lue une adresse des pensionnaires du couvent du Cap, demandant a entrer dans leurs familles. Elles accusaient les Religieuses de ne savoir mieux les édifier que les instruire. La lettre était anonyme, signé des initiales L.L.L.L. L. "pour toutes les Sœurs qui craignent également le despotisme clôtral".

"L'Assemblée arrêta que l'Assemblée provinciale du Nord, siégeant au Cap enverrait des commissaires dans le couvent a l'effet d'empêcher la Supérieure, et que las unes et les autres seraient interrogées à ce sujet. Le fond de la question fut ajourné à huit jours". ${ }^{44}$

Jamais la question ne fut traite à fond; il est probable d'ailleurs que l'Assemblée du Nord ne remplît même pas son mandat, car elle ne tarda pas à se brouiller avec l'Assemblee de Saint-Marc; aussi bien l'Assemblée de Saint-Marc fut bientôt occupée d'affaires plus importantes que ne l'était la protestation de pensionnaires de couvant, et nous verrons que deux ans plus tard la maison des Religieuses avait encore conservé quelques esprits chagrins qui y mettaient le trouble. Des faits plus graves intervinrent.

Pour les exposer, nous n'avons plus le récit original qu'en ont fait les Religieuses dans leur correspondance. Nous ne possédons que l'histoire de la Communauté écrite par un chroniqueur d'après cette correspondance sans doute, mais correspondance complétée et inter-

44. Archives de la Seine et Oise. Papiers Kessaint. E 1453. Journal des séances d e l'A ssemblée coloniale de Saint-Marc (15 mai - 2 juillet 1790). 
prété par des relations d'historiens contemporains; ces historiens ont eux-mêmes usé de connaissances révélées par des événements postérieurs, en sorte que le chroniqueur qui les suit attribue aux Religieuses des notions que celles-ci ne pouvaient avoir: nous tâcherons de retrouver à travers sa narration ce qu'il a extrait des lettres des Religieuses.

Les Religieuses, depuis quelques années, consta taient dans la ville du Cap, parmi les blancs un engouement jusque là inconnu pour la franc-maçonnerie; elles furent choquées par une parole de prêtre qui au service anniversaire célébré pour la Mère de Lavaud, fit l'éloge de la défunte: 'Non, frères et amis, avait dit l'orateur, elle n'est pas morte; elle s'est envolée sur les ailes séraphiques des Chérubins, dans l'Elysée où les philanthropes reposent dans le sein du Grand Architecte!" Les femmes, les jeunes filles créoles s'enrôlaient dans les sociétés secrètes; les clubs politiques trouvaient leur force et leur lien dans ces réunions clandestines. Peut-être aussi, à l'exemple des blancs, les noirs avaient-ils leurs conciliabules apparentés au Vaudoux; mais il importe de $r$ marquer que les allusions au Vaudoux sous la plume des Religiauses seraient un anachronisme avant le mois d'aout $1792 . \mathrm{Si}$ le Vaudoux eat une influence qu'on ne peut nier sur les événements de ce temps, il n'avait, aux yeux des blancs, qu'une assez mince importance; on n'y voyait qu'une manifestation bénigne de la mentalité des esclaves, une sorte de folklore sans grande portée sociale.

Il paraît certain pourtant que peu avant les événements du mois d'aout 1791, on ait été porté à voir comme une sorte de franc-maçonnerie des noirs dans certaines manifestations de leur activité. Parmi les jeunes filles noires, anciennes élèves des Religieuses, une effervescence insolite se marquait par des danses, par des refrains inusités qu'on supposait être des chants nègres. Celle qui semblait à la tête de ce mouvement se nommait Améthiste, que le chroniqueur identifie avec la fille de Henri Christophe, ce qui n'est pas vraisemblable. Un soir, plusieurs de ces filles sortirent de la ville at se répandirent dans la plaine, ceintes à la taille de mouchoirs rouges; les Religieuses avaient remarqué cet accoutrement jusque chez leurs élèves.

Cette surexcitation s'expliqua quand, à la fin d'août 1791, on se trouva au Cap en face du soulèvement de Boukman, des incendies des habitations aux abords de la ville et de la dévastation de la plaine. De chez elles, les Religieuses virent les insurgés, aux portes du Cap; elles entendirent leurs cris de mort, elles furent témoins de leurs dan 
ses; elles ressentirent la terreur qui avait envahi l'âme des blancs à la nouvelle des massacres accomplis dans la campagne et des ruines qui s'y accumulaient.

Leur situation devenait angoissante. Quel serait, en effet, leur avenir et celui de leur communauté et de leur œuvre? De France, elles n'avaient plus à attendre aucun secours puisque les Ordres Religieux, les Congrégations ecclésiastiques ou laĩques avaient été supprimés et le port de l'habit religieux interdit (5 avril 1791). Sous leurs yeux, les Capucins, chargés de la Mission, avaient obtenu l'indult \& sécularisation et en jouissajent. On leur apprenait de France que la plupart des religieux rentraient dans le monde, qu'un très petit nombre acceptaient la vie en commun avec des membres d'autres Ordres arrachés, eux aussi, à leur observance propre. Elles agitèrent la question de l'abandon de leur couvent et de leur retour en France. Vivement sollicitées de rentrer dans leurs familles afin de mettre leurs personnes en sûreté, elles choisirent de rester à leur poste au service des filles noires et de couleur et ne cessèrent de les instruire et de les assister. Leur courage enhardit quelques-unes de leurs grandes pensionnaires qui restèrent près d'elles et le calme se rétablit.

Le 21 septembre 1792 arriva au Cap une Commission Civile c'était la seconde - pour administrer le pays au nom de l'Assemblée législa tive qui l'avait nommée, puis au nom de la Convention Nationale qui succéda en septembre 1792 à la Législative. Les deux principaux membres en étaient Sonthonax et Polverle. Sonthonax, le plus jeune, prit vite le pas sur son collègue par les mesures extraordinaires qu'il décréta. C'était un de ces hommes de la Révolution, sans expérience des affaires, guidé souvent par des idées généreuses qu'il imposait parfois par des moyens excessifs et même révoltants. A Saint-Domingue il se posa en réformateur et travailla à abolir un passé qu'il jugeait inique. Il s'entremit même entre les Religieuses et leurs pensionnaires pour leur prêcher la paix et la concorde au nom de Jésus-Christ (12 novembre 1792), nouvel incident du conflit soulevé deux ans plus tôt et soumis à l'Assemblée de Saint-Marc.

Aux journées de juin 1793, Sonthonax, pour vaincre le général Galbaud, gouverneur, lâcha sur le Cap les bandes insurgées des noirs qui mirent la ville à feu et à sang. Les Religieuses furent d'abord défendues par d'anciens esclaves reconnaissants des bienfaits qu'ils avaient reçus d'elles. On ne leur en voulait pas, mais on poursuivait 
leurs pensionnaires; malgré leurs protestations, leur maison fut enfin envahie. Les filles blanches furent massacrées après d'horribles tortures.

Quant aux Religieuses qu'on mentionne au nombre de six, elles tentèrent de se réfugier a bord des vaisseaux en rade avec la plupart des blancs. Les deux Mères Goursaud y réussirent; les autres, les Mères Cases, de Laborderie et Minard furent comptées parmi les victimes; on ne parle pas de la Mère Marais qui, créole du Cap, réussit sans doute à échapper aux poursuites.

La frégate LaFine, qui portait les deux Mères Goursaud, fut, au voyage de retour en France, prise sans combat par les Anglais. La Mère Goursaud de Saint-Joseph, nous ne savons pour quel motif, fut pendue par l'ennemi aussitôt après la reddition du navire; seule, sa sœur, la Mère Goursaud de Bonnefond parvint en France; elle était accompagn6e d'une jeune fille noire, Suzanne Louverture qui n'avait pas voulu se séparer de ses maitresses et qui mourut à l'hôpital de Limoges le 17 février 1815.

Là s'arrête dans les traditions de l'Ordre l'histoire des Religieuses du Cap Français. D'autres souvenirs ont survécu. Une Américaine, Miss Hassal, qui a vécu au Cap pendant la guerre de l'Indépendance d'Haìti, a laissé des Lettres ${ }^{45}$ traduites en 1936 par M. Pauléus Sannon. ${ }^{4} 6$ Elle fait mention du couvent du Cap.

Les nonnes en général étaient très riches et se consacraient principalement à l'education des jeunes filles; quelques-unes de leurs élèves auraient fait honneur à un couvent parisien, d'après ce que j'ai entendu dire.

Quand la religion fut abolie en France, la rage anti-religieuse autant que la rage révolutionnaire gagnèrent cette ville (du Cap) et les nonnes furent chassées de leur couvent par Sonthonax, un nom qui remplira toujours d'horreur le coeur de tout Français...

A l'arrivée du général Galbo (Galbaud) que l'on avait envoyé pour le remplacer, il dit: "Si Galbo règne ici, il régnera sur les cendres" et mit le feu effectivement à la ville. Le couvent ne fut pas alors brûlé, mais la communauté fut dissoute, l'habit de l'Ordre mis de côté et quelques religieuses profitant du relâchement des mœurs se marièrent. "L'une d'elles devint la femme d'un homme qui, pendant le règne des nègres commit les crimes de la plus grande noirceur. Il n'a pas encore reçu la punition due à ses forfaits; mais il attend en tremblant le jour du châtiment.

45. Secret History or the Horrors of St. Domingo in a series of letters, written by a lady at Cape François to colonel Burr, late vice-president of the United States principally during the command of general Rochambeau. (Philadelphia, 1802).

46. Le Cap Français vu par une américaine. (S.L. 1936), in-12, 78. 
Je la vois souvent. Elle a été très belle, mais ses charmes sont maintenant à leur déclin. Elle a beaucoup de vivacité et de facilité d'élocution, en s'entretenant des choses du jour, ce qui donne à la française la réputation d'avoir beaucoup d'esprit.

Je connais aussi l'abbesse qui est une excellente femme aux manières les plus engageantes. Elle vit dans une miserable chambre et gagne sa vie par son travail.

La plus grande partie de la communauté périt et le géneral Leclerc trouva plus convenable de réparer le couvent et d'en faire sa propre résidence - la maison du gouvernement ayant été entièrement détruite, plutôt que de la rendre à ses propriétaires.

Ce témoignage ne saurait être contesté dans ses principales assertions. Nous ignorons pourtant quelle est cette religieuse maríe. Les traditions qui rapportent le massacre des trois Mères Cases, de Laborderie et de Minard sont peut-être établies sur l'absence de tout renseignement à leur égard; il pourrait se faire aussi que la Maison ait reçu dans ses dernières années quelque novice de la Colonie.

Un autre témoignage ou une autre tradition, rapporté par Pamphile Lacroix ${ }^{4} 7$ assure qu'en 1799 Toussaint Louverture arrivé inopinément au Cap tint une grande réunion chez lui où "il interrogea de jeunes personnes blanches qui lui furent présentées par une religieuse chargée de leur éducation". On penche à croire qu'il s'agit de cette mère que Miss Hassal appelle l'abbesse. Serait-ce la mère Marais?

Il est certain qu'en 1802 on connaissait encore la supérieure, et il est vraisemblable qu'elle était originaire du Cap. Mais nous ne savons pas les noms de toutes les religieuses du Cap.

Il en est une qui survécut. Monseigneur Hillion a gardé son souvenir dans les Relations de ses Visites pastorales:

"Pendant la guerre de l'Indépendance, dit-il, cet établissement (la Providence des Femmes) fut presque détruit. Le roi Christophe le fit restaurer et en confia la direction à une ancienne Religieuse qui avait échappé au massacre des Blanes, à Madame Saint-Martin, dont la charité a laissé dans la population du Cap un reconnaissant et religieux souvenir. Elle a 6 te enterree dans la chapelle (des Religieuses) entre la porte de la sacristie et la balustrade qui ferme le sanctuaire. Sur la pierre tombale en marbre qui recouvre ses restes mortels, on a gravé cette inscription: "Ci-gît Jeanne Germaine Saint-Martin, religieuse, décédée le 6 novembre 1822, à l'âge de 71 ans et 7 jours.

47. Mémoires pour servir à l'histoire de la Révolution de Saint-Domingue, (2 vol., Paris, 1819), I: 379.

48. Le nom de la ville indique le couvent d'origine des religieuses. 
Elle était vraisemblablement originaire de Saint-Domingue où les Saint-Martin avaient tenu de hautes fonctions; l'un d'eux fut longtemps président du Conseil du Cap.

Ainsi s'achève, après 90 ans, l'histoire de cette Communauté des Filles de Notre-Dame du Cap Français. Le P. Boutin avait voulu d'abord une maison d'Hospitalières; il eut des Religieuses enseignantes et la dernière d'entre elles porte dans un hôpital restauré le renom traditionnel de la charité des Congrégations françaises. 